\title{
Genome of Bifidobacteria and Carbohydrate Metabolism
}

\author{
Pauline Bondue and Véronique Delcenserie* \\ Fundamental and Applied Research for Animal \& Health (FARAH), Food Science Department, \\ Faculty of Veterinary Medicine, University of Liège, Sart-Tilman, B43b Liege, B-4000 Belgium.
}

\begin{abstract}
In recent years, the knowledge about bifidobacteria has considerably evolved thanks to recent progress in molecular biology. The analysis of the whole genome sequences of 48 taxa of bifidobacteria offers new perspectives for their classification, especially to set up limit between two species. Indeed, several species are presenting a high homology and should be reclassified. On the other hand, some subspecies are presenting a low homology and should therefore be reclassified into different species. In addition, a better knowledge of the genome of bifidobacteria allows a better understanding of the mechanisms involved in complex carbohydrate metabolism. The genome of some species of bifidobacteria from human but also from animal origin demonstrates high presence in genes involved in the metabolism of complex oligosaccharides. Those species should be further tested to confirm their potential to metabolize complex oligosaccharides in vitro and in vivo.
\end{abstract}

Key words: bifidobacteria, genomic, complex oligosaccharides, inuline, galacto oligosaccharides, human milk oligosaccharide, bovine milk oligosaccharide

\section{Introduction}

In 1899, Tissier was the first person to isolate bifidobacteria from feces of breast-fed children. Because of their "bifides" Y-shape, and because their physiology is similar to lactobacilli, they have first been named Bacillus bifidus and then classified in the genus Lactobacillus. It was finally in 1974 with the $8^{\text {th }}$ edition of Bergey's manual, that bifidobacteria were classified in a different genus, the genus Bifidobacterium. This genus belongs to the family of Bifidobacteriaceae, belonging to the order of Bifidobacteriales and is one of the branches of the phylum Actinobacteria. This phylum includes both pathogens such as Mycobacterium spp. but also bacteria beneficial to our health, such as bifidobacteria. The bacteria included in this phylum have physiological and metabolic properties as varied as their morphologies are different. The genus Streptomyces is the most studied taxon of this phylum as its metabolites have antibiotic properties used in the pharmaceutical industry (Di Gioia et al., 2014; Turroni et al., 2011; Turroni et al., 2014).

\footnotetext{
*Corresponding author: Véronique Delcenserie, Food Sciences Department, Faculty of Veterinary Medicine, University of Liege, Sart-Tilman, B43b Liege, B-4000 Belgium. Tel: +32-4366-51-24, Fax: +32-4-366-40-44, E-mail: veronique.delcenserie@ulg.ac.be
}

Bifidobacteria are Gram-positive saccharolytic bacteria whose genome is rich in guanine and cytosine $(G+C)$. The vast majority of bifidobacteria are strict anaerobes, but some of them are microaerophilic such as $B$. psychraerophilum, B. crudilactis and B. mongoliense. They present fermentation metabolism (De Vuyst et al., 2013; Turroni et al., 2014) but they do not produce gas. They are non-spore forming and non-motile bacteria (Bottacini et al., 2014; Sela and Mills, 2010).

Bifidobacteria specifically degrade hexoses monosaccharide (glucose and fructose) using the "fructose-6phosphate phosphoketolase pathway", also called bifid shunt. They are unable to classically ferment the hexoses since they have neither the aldolase neither phosphofructokinase, (present in homo-fermentative lactic acid bacteria) or glucose-6-phosphate dehydrogenase (present in hetero-fermentative lactic acid bacteria). Therefore, they are unable to ferment hexose monosaccharides through the Emden-Meyerhof-Parnas (EMP) pathway or 6-phosphogluconate/phosphoketolase pathway, respectively. However, with the bifid shunt and the production of a specific enzyme: the fructose-6-phosphate phosphoketolase (F6PPK), they are able to produce more ATP from glucose and fructose.

From the monosaccharides of two hexoses, there is a formation of three moles of acetate, two moles lactate and five moles of ATP. In theory, the acetate: lactate ratio is 
$3: 2$. In reality, this ratio is rarely observed as pyruvate can be metabolized in acetate, formate or even ethanol (De Vuyst et al., 2013). If a large amount of carbohydrates is available for the growth of bifidobacteria, a large amount of lactate will be generated compared to acetate, formate or ethanol. On the opposite, if the cell survival is threatened, because the source of energy is less available, the carbohydrates will be consumed slowly and lactate production will be lower compared to a higher production of acetate, formate and ethanol. This enables the bacteria to produce and save more energy when a lot of substrate is available. In the colon, the exogenous acetate produced by bifidobacteria can be metabolised by butyrate-producing bacteria (De Vuyst et al., 2013). Therefore, the butyrogenic effect caused by butyrate-producing colon bacteria could rather be due to cross-feeding interactions rather than direct fructan consumption.

Bifidobacteria are also able to produce small amounts of succinic acid, as demonstrated by Van der Meulen et al. (2006) on 10 strains of bifidobacteria belonging to different species. This production seems to play a role in the regeneration of NAD+ occurring through the production of lactic acid or ethanol.

Excellent studies and literature reviews were recently published about Bifidobacterium genus. The aim of this review is to report those recent advances in molecular biology and microbiology allowing new classification of species inside the Bifidobacterium genus. In addition, an update about the known metabolism involved in catabolism of several complex oligosaccharides by those bacteria is given.

\section{Genotypic Characteristics and Taxonomy: Focus on New Species}

Our knowledge of bifidobacteria has considerably evolved in recent years. Based on the current scientific knowledge, the genus Bifidobacterium consists today of 48 official taxa representing 39 species and 9 subspecies (Lugli et al., 2014). Forty taxa were isolated from the gastrointestinal tract of mammals, birds, or insects (Arrieta et al., 2014), and eight were isolated from sewage, fermented milk or blood. As indicated by previous studies involved in gastrointestinal microbiota analysis, additional bifidobacterial species are to be discovered in the future (Turroni et al. 2012; Zoetendal et al., 2008) and further expansion or refinement of the classification is to be expected.

Based on 16S rRNA sequences data from all recognized species (1265 nucleotides), a tree highlighted 3 new phylogenetic clusters groups, B. crudilactis, B. bohemicum and $B$. scardovii groups, in addition to the usual six, representing: $B$. adolescentis, $B$. asteroides, $B$. boum, B. longum, B. pullorum and B. pseudolongum (Lugli et al., 2014; Turroni et al., 2014).

Based on 23S rRNA sequences, the analysis of the 48 taxa confirmed the classification into the six usual previously recognized groups.

However, the study of phylogeny within a specific microbial taxon using only one molecular marker is limited today considering the tremendous progress in molecular biology. In addition, 16S rRNA sequences can be of high similarity for two different taxa. Several alternative approaches were described using 16S rRNA gene associated with other genes. Analysis of identity levels based on 16S rRNA and 23S rRNA sequences showed that 19 bifidobacterial pairs still present more than $97 \%$ of identify, the threshold usually considered for species separation (Lugli et al., 2014; Stackebrandt et al., 2002). An approach employing concatenation 16S rRNA gene with six other housekeeping genes $(c l p C, d n a J, x f p, d n a B$, rро $C$ and $p u r F$ ) for sequence analysis was described (Ventura et al., 2006). This approach allowed a significant increase of discrimination between taxa. Another approach according to Deletoile et al. (2010) based on concatenated sequences of the housekeeping genes $\operatorname{clp} C$, fus $A$, gyrB, ileS, purF, rplB and rpoB, and performed on 11 species allowed the classification of $B$. crudilactis and $B$. mongoliense in two different clusters, well separated from the other bifidobacteria clusters (Delcenserie et al., 2013).

Recent progress in molecular biology offer now the possibility to define an Average Nucleotide Identity (ANI) using whole genome sequencing (Konstantinidis and Tiedje, 2005). The whole genome sequences of the 48 taxa of bifidobacteria have been studied, and are, today, the most complete genome analysis of those bacteria. They allowed the identification of 18,435 bifidobacterium-specific clusters of orthologous genes (bifCOGs). Analysis of these bifCOGs allowed the identification of 534 COGs thereby forming the heart of the sequences encoding the genome of bifidobacteria (core bifCOGs). With the sequences of the genomes, it was possible to identify a set of 411 genes that were then used to build a new phylogenetic tree for bifidobacteria, named "supertree". That tree highlighted 7 phylogenetic groups instead of the usual six, with the appearance of a new cluster representing $B$. bifidum group (Lugli et al., 2014). The ANI was also obtained for each bifidobacterial pair and varied from $81.3 \%$ to $98.1 \%$ of identity. As suggested by Lugli et al. (2014), 
ANI could be used instead of DNA-DNA hybridization, the gold standard, to set up the limit between two different species. Interestingly, using ANI, the taxonomy of bifidobacteria would be refined with the recognition of only 34 species of bifidobacteria instead of 39. Indeed, some pairs presented a ANI of $97 \%$ such as B. stercoris, $B$. coryneforme, $B$. kashiwanohense and $B$. saeculare comparing to respectively $B$. adolescentis, $B$. indicum, $B$. catenulatum and $B$. gallinarum/B. pullorum). On the other hand, the $B$. pseudolongum subsp. pseudolongum and $B$. pseudolongum subsp. globosum pair presented an ANI below $94 \%$ and should therefore be reclassified into two different species. Other subspecies were confirmed with ANI between 94 and 97\% (Lugli et al., 2014).

The genome size of bifidobacteria chromosomes varies from 1.9 to $2.8 \mathrm{Mbp}$. B. animalis has the smallest genome and $B$. longum subsp. infantis has the largest. An average of 2,012 open reading frames (ORFs) is observed per genome. B. animalis subsp. lactis and B. longum subsp. infantis are the species having the lowest and the most ORF, respectively (Bottacini et al., 2014). The genome of bifidobacteria adapts easily to new environments. Largescale commercial use has created new media very rich in nutrients and, for example, $B$. animalis subsp. lactis has adapted to these environments by losing genes. The genome evolves over time through different mechanisms: gene duplication, chromosomal rearrangements and DNA vertical and horizontal intraspecies gene transfer (Bottacini et al., 2014).

The mobilome corresponds to all the mobile parts. It is characterized by insertion sequences, bacteriophage element, prophage-like elements or plasmides. The most abundant and active insertion sequences are the IS30 elements. Those elements are responsible for deletions and rearrangements into the genome and are involved in rapid environmental adaptation of bifidobacteria, for example an adaptation on a culture media (Lee and O'Sullivan, 2010).

Bacteriophages and prophage-like elements are involved in lateral gene transfer. Apart from the strains of $B$. longum subsp. longum and B. breve, bifidobacteria do not contain plasmids (Bottacini et al., 2014).

Bifidobacteria are present in many ecological niches. It is found in the human and animal intestinal tract (bovine, rabbit, mice, chicken and insect), in the oral cavity, in stagnant water and in food. As lactic acid bacteria, they are often added to fermented foods, such as yoghurt. However, the vast majority of bifidobacteria species is only able to grow in the absence of oxygen, a condition difficult to reproduce in industry. Some of them can survive the acidity of the stomach environment, duodenal, bile salts and pancreatic juices but those are conditions usually aggressive for bifidobacteria.

They are transmitted from mother to infant by direct vertical transmission in most cases, but also indirectly by contamination of the environment. Some bifidobacteria have a cosmopolitan lifestyle (B. animalis, $B$. adolescentis) while others are adapted to the digestive ecology of a specific animal species. For example, $B$. cuniculi is encountered in rabbit, $B$. gallinarum in chicken and $B$. pseudolongum in bovine (Turroni et al., 2011). Bifidobacteria adapted to a specific ecosystems can be excellent indicators of fecal contamination and can be considered as being more effective as currently used indicators such as coliforms. For example, B. pseudolongum isolated from raw milk cheese industry, indicates fecal contamination of bovine origin and not from human origin because this species is not encountered in the human intestinal tract (Delcenserie et al., 2011). As mentioned earlier, the predominant species found in humans vary with age, and this because of their genome. In a child, predominant species are $B$. bifidum and $B$. longum subsp. infantis while in adults it is $B$. adolescentis and B. longum subsp. longum. Genes involved in carbohydrate metabolism are responsible for changing this distribution, in particular the genes coding for carbohydrate transporters and for glycosidases, mainly $\beta$-galactosidase and $\beta$-fructofuranosidases (De Vuyst et al., 2011). The majority of bifidobacteria encountered in children have a genome oriented in the degradation of human milk oligosaccharides (HMOs), while those of adults have a genome oriented toward degradation of dietary carbohydrates. Degradation of complex carbohydrates, development of secondary metabolites and the production of acetate and lactate through the Bifid Shunt are based on interactions between bifidobacteria and other microorganisms present in the intestinal tract (De Vuyst et al., 2011). For example, Bacteroides and other gut bacteria secrete various glycosidases able to metabolize complex sugars (Sela and Mills, 2010). Twelve percent of the genome of bifidobacteria is dedicated to transportation of carbohydrates or their metabolism, while $5 \%$ of the genome is dedicated to protein metabolism (Sela and Mills, 2010).

\section{Carbohydrate Fermentation Pattern of Bifidobacteria}

\section{Inuline and fructooligosaccharides (FOS)}

Inulin is composed of long chain of fructose. The deg- 
ree of polymerisation (DP) of the inulin is 10 on average but can range from 3 to 60 . FOS are composed of the same fructose found in inulin, but the degree of polymerization range around 4 and can vary from 2 to 8 . The higher is the degree of polymerization, the longer the fermentation will be. Thus the shortest OS will be consumed first while the longest will be gradually consumed (Bosscher et al., 2006). Inulin is extracted from chicory roots (Cichorium intybus) where it is present in more than $70 \%$ in dry matter. Other foods naturally rich in inuline or FOS include onions, artichokes, asparagus, leeks and cabbage (Rossi et al., 2005). The oligofructose or FOS, are formed by partial hydrolysis of inulin by fructanases and sucrases (Chichlowski et al., 2011). They can also be produced by transfructosylation from fructose. Inulin and FOS arrive intact in the colon as they are neither absorbed nor degraded due to their $\beta$ bonds (1-2). The fermentation of carbohydrates takes place in the proximal part of the colon, which is highly saccharolytic comparing to the distal portion, which is more proteolytic. Fermentation of inulin and FOS by lactobacilli and bifidobacteria produces organic acids such as lactate and succinate that can exert antimicrobial effect against pathogenic bacteria by reducing their growth, spread and passage into the bloodstream, while promoting the growth of beneficial bacteria and thus the defenses of host. In addition, thanks to its high degree of polymerization, inulin is able to reach the distal parts of the colon where it is preferentially proteolysis, and is able to reduce the latter in favour of a more beneficial saccharolytic activity (Bosscher et al., 2006).

Some strains of bifidobacteria are able to consume oligofructose and inulin through a $\beta$-fructofuranosidase activity. Some strains are able to degrade small oligosaccharides by intracellular degradation (Fig. 1(a)). This confers a competitive advantage to those strains against inulintype fructan degraders such as lactobacilli, bacteroides and roseburia. Some other strains are able to metabolize long chain oligofructose and inulin. A recent study (Stiverson et al., 2014) demonstrated that a commercial formulation of long chain inulin (Beneo ${ }^{\circledR}$ Synergy 1$)$ exerted a bifidogenic effect by increasing total bifidobacteria and more specifically $B$. longum in infant microbiota. A study performed with pig fecal microbiota showed that long chain inulin increased total bifidobacteria (Han et al., 2014). In this study, it was suggested that animal bifidobacterial strains were better able to metabolize inulin than human strains, especially long chain inulin (Average DP of 25).

Fermentation of inulin and FOS produces short chain fatty acid (SCFA), lactic acid and gas (carbon dioxide and hydrogen). The produced SCFA such as acetate, propionate and butyrate are either used by the intestinal microbiota, or used as an energy source by the host. Inulin and FOS are able to provide 1.0 or $1.5 \mathrm{kcal} / \mathrm{g}$, respectively (Bossher et al, 2006). Inulin fermentation provide mainly butyrate, while FOS provide preferentially acetate and lactate. SCFA, especially butyrate, have an important role in preventing colon cancer (Rossi et al., 2005). However, as mentioned earlier, the production of butyrate is not due to direct metabolism of inulin by bifidobacteria but rather by cross-feeding interaction with butyrate-producing colon bacteria, such as clostridia (De Vuyst et al., 2013). Two types of cross-feeding have been identified, the first concerns mono or oligosaccharides formed by bifidobacteria during degradation, while the second is the organic acids such as lactate and acetate (De Vuyst and Leroy, 2011).

\section{Galacto-oligosaccharides (GOS)}

Galacto-oligosaccharides (GOS) are synthetized from lactose and $\beta$-galactosidase. The main component of GOS is 4'-galactosyl-lactose, which consists of one galactose bonded to lactose through galactose $\beta 1-3 / 4 / 6$ linkages. The polymerization degree ranges from 3 to 15 and there is a terminal glucose (Garrido et al., 2013). GOS are widely used for their bifidogenic properties. However, other species such as Bacteroides sp. and Clostridium $s p$. are likely to metabolism GOS too.

In commercial formulations for infants, GOS are often mixed with FOS. In previous described studies, the mixture was composed of $90 \%$ GOS short chain and $10 \%$ long chain FOS (inulin having DP> 23). With this ratio, an increase in the number of bifidobacteria was observed in infant feces. In parallel, a decrease in the incidence of pathogens such as E. coli, Enterococcus and an improvement of transit time and stool consistency was observed (Chichlowski et al., 2011; Scholtens et al., 2014). A recent study showed that GOS alone or in combination with inulin were able to support the growth of $B$. longum and to induce a strong antimicrobial effect against E. coli, mainly due to the production of acetic acid (Stiverson et al., 2014). A study performed with a gastrointestinal model for pig demonstrated that B. thermophilum RBL67 was able to metabolize GOS and produce antimicrobial effect against Salmonella Typhimurium through the production of acetate (Tanner et al., 2014). Finally, another study showed the potential of $B$. breve 46 to metabolize GOS in a gastrointestinal model comparing to $B$. 
Glucose

Galactose

N-acetylglucosamine

Aucose

- Sialic acid

Fructose

$\alpha \quad 1-2 / 3 / 4$ bound

$\alpha 2-3 / 6$ bound

$\beta 1-3 / 4 / 6$ bound

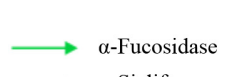

$\longrightarrow \alpha$-Sialifase

$\longrightarrow \beta$-Hexosaminidase

$\longrightarrow \beta$-Galactosidase

Lacto-N-biosidase

$\longrightarrow$ LNB phosphorilase

$\longrightarrow \beta$-Fructofuranosidase

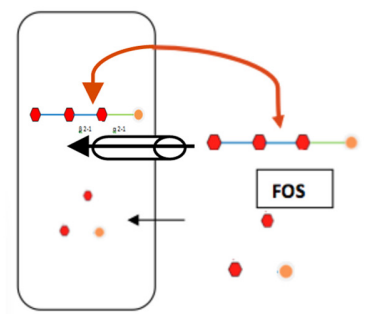

B. bifidum

B. longum subsp.

infantis

B. breve

Other

bifidobacteria

from animal origin

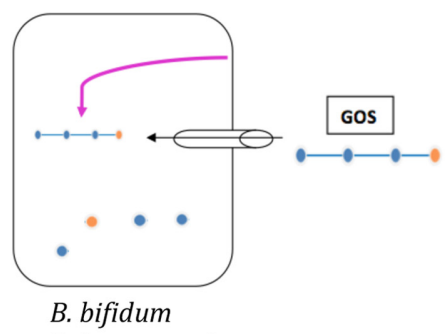

B. bifidum

B. longum subsp.

infantis

B. breve

B. thermophilum

a.
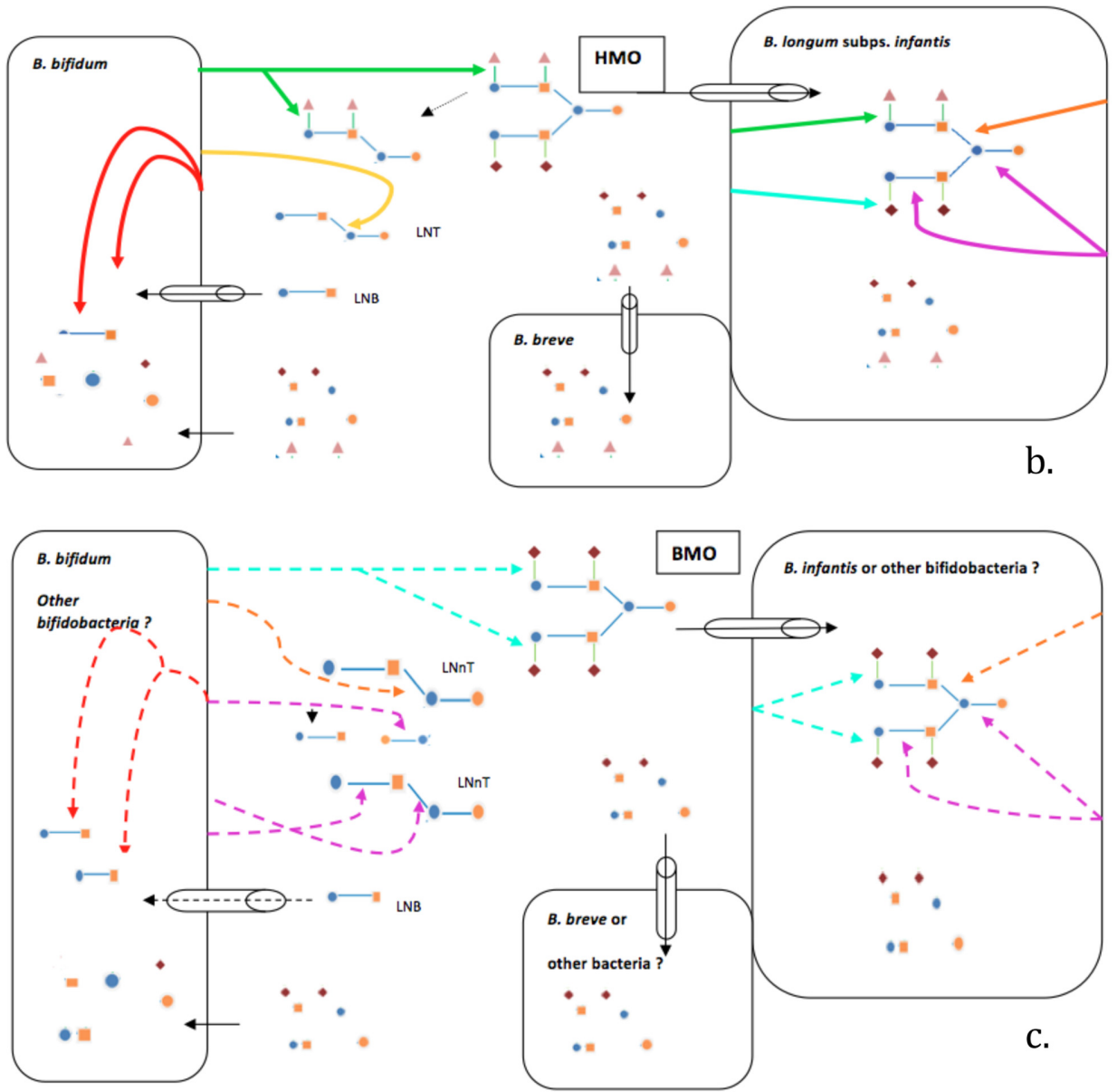

Fig. 1. A schematic representation of the degradations patterns of complex oligosaccharides by bifidobacteria. Inspired from Smilowitz et al., 2014, Garrido et al., 2012, Cardelle-Cobas et al., 2011, Zivkovic and Barile, 2011, Sela and Mills, 2010, Boscher et al., 2006. (a) Degradation of FOS or inulin by bifidobacteria using a $\beta$-fructofuranosidase activity. Degradation of GOS by bifidobacteria using specific carriers and $\beta$-galactosidase activity. (b) Degradation of HMO by Bifidobacterium longum subsp. infantis using transport systems, intracellular glycosyl hydrolase and $\alpha$-fucosidase, $\alpha$-sialidase, $\beta$ galactosidase and $\beta$-N-hexosaminidase. Degradation of HMO by Bifidobacterium bifidum using lacto-N-biosidase, $\alpha$ fucosidase, $\alpha$-sialidase and LNB phosphorilase. Use of extracellular metabolized HMOs by $B$. breve and $B$. longum subsp. longum. (c) Hypothetic degradation of BMO by bifidobacteria using mainly sialidase activity. Both potential pathways involving either intracellular or extracellular degradation are represented. FOS, fructooligosaccharides; GOS, glucooligosaccharides; HMO, human milk oligosaccharides; BMO, bovine milk oligosaccharides; LNB, lacto-N-biose; LNT: lacto-N-tetraose; LNnT: lacto-N-neotetraose. 
pseudocatenulatum JCM1200 or B. animalis subsp. lactis 8:8 (Adamberg et al., 2014).

To degrade GOS, bifidobacteria should be able to synthetize specific carriers and $\beta$-galactosidases (Fig. 1(a)). For example, B. longum subsp. infantis has 5 genes encoding $\beta$-galactosidases, 3 of them being involved in metabolism of GOS. It preferentially consumes GOS with a polymerisation degree of 4 , but all subspecies, due to phenotypic differences, will not consume the same way (Garrido et al., 2013). In addition, the degree of polymerization and the connection types influence its degradation and the speed of degradation too (Cardelle-Cobas et al., 2011).

\section{Human milk oligosaccharides (HMO) and bovine milk oligosaccharides (BMO)}

\section{Human milk oligosaccharides (HMO)}

The formation of HMO occurs in the mammary gland with $\beta$-galactotransferase in the presence of $\alpha$-lactalbumin. Glucose and galactose, linked by a $\beta 1-4$ linkage form the so-called lactose heart. HMOs are formed from 5 monosaccharides: glucose, galactose, $\mathrm{N}$-acetylglucosamine, fucose and sialic acid. They may be linear or branched and include from 3 to 14 monosaccharides. The terminal lactose can be linked to a fucose (through $\alpha-1,2$, $\alpha-1,3$ or $\alpha-1,4$ liaisons) or sialic acid (through $\alpha-2,3$ or $\alpha$ 2,6 liaisons). This terminal lactose can be elongated by a lacto-N- biose type 1 or $\mathrm{N}$ - acetyllactosamine. In human milk, a recent estimate fixed fucosylated HMO to 35 to $50 \%$, sialylated to 12 to $14 \% \mathrm{HMO}$ and 42 to $55 \%$ as neutral nonfucosylated HMO. In the presence of negatively charged sialic acid, HMO is qualified as acid. Otherwise it is called neutral (Smilowitz et al., 2014).

HMO concentration reaches $25 \mathrm{~g} / \mathrm{L}$ in colostrum and 10 to $15 \mathrm{~g} / \mathrm{L}$ in mature milk. More than 200 different structures have been identified so far (Mehra et al., 2014). Because of their bifidogenic properties, HMOs play an active role in the development of a beneficial microbiota in infant by allowing the growth of specific bacteria. In addition, it is described that HMOs increase the immune defenses of the host. One of the mechanism is that fucosylated HMOs present similarity with surface receptors of the mucous layer in the intestinal tract and therefore bind to pathogens, allowing them to be washed out naturally (Smilowitz et al., 2014). The sialylated HMOs are also involved in brain and cognitive development of the child (Wu et al., 2011). HMOs are protected from human digestion through their terminal sialylated and fucosylated chains. Therefore, bifidobacteria must have clusters of genes encoding specific enzymes and carriers to be able to digest these HMOs. It has been described that those gene clusters are located on mobile parts (Sela, 2011). In addition, there is a co-evolution between HMO present in the microbiota and bifidobacteria able to use them (German et al., 2008). More recently, it has been described that some bifidobacteria clusters are more particularly adapted for HMO catabolism (Milani et al., 2014). Indeed, that cluster seems to have lost genes encoding glycosyl hydrolases involved in the degradation of plant polysaccharides.

B. longum subsp. infantis preferentially metabolize small HMO (degree of polymerization of less than 8 and molecular weight of less than $1400 \mathrm{Da}$ ), which represent $64 \%$ of the available HMO (Sela and Mills, 2010). It is able to grow in a medium containing HMO as a sole carbon source. With its gene cluster of $43 \mathrm{~kb}$ which encodes transport systems and intracellular glycosyl hydrolases, $B$. infantis possesses genes coding for 4 enzymes necessary for digestion of HMO: $\alpha$-fucosidase, $\alpha$-sialidase , $\beta$ galactosidase and $\beta$-N-hexosaminidase. This bacterium possesses as well genes of $\mathrm{ABC}$ transporters and their associated specific binding proteins (SBP). B. infantis internalizes the HMO and only then exposes the degraded lactose heart deprived of its fucose or sialic acid (Sela et al., 2008; Sela, 2011; Smilowitz et al., 2014).

On the opposite, the digestion of HMO by B. bifidum is an extracellular process. The cluster of genes present in B. infantis is absent from the genome of B. bifidum and different enzymes are synthetized: lacto-N-biosidase in addition to sialidase and fucosidases. These separate LNB (lacto-N-biose) from HMO. It passes through the membrane, with an $\mathrm{ABC}$ transporter associated with a specific SBP, and is then degraded intracellularly (Sela, 2011; Smilowitz et al., 2014). Interestingly, B. bifidum and B. infantis grow relatively well in HMOs, but have distinct clusters of genes involved in their metabolism (Barile and Rastall, 2013). A schematic representation of the degradations patterns is presented in Fig. 1(b).

Interestingly, unlike $B$. longum subsp. infantis that has all the necessary genes to digest HMO and is consequently highly represented in the microbiota of a breastfed child, B. longum subsp. longum (part of the majority bifidobacteria microbiota in adult) is unable to metabolize HMO (De Vuyst et al., 2013). However, there is a close cooperation in the metabolism of HMO between different bifidobacteria. B. longum subsp. longum, B. bifidum and $B$. breve are able to metabolize lacto-N-biose obtained 
after HMO metabolism by $B$. infantis. LNB can be metabolized through the action of fucosidases and lacto-Nbiosidases in an extracellular process. Monosaccharides obtained from successive HMO degradations are mainly used by B. breve (Barile and Rastall, 2013; Sela and Mills, 2010).

\section{Bovine milk oligosaccahrides (BMO)}

The concentration of BMO in bovine colostrum range from 0.7 to $1.2 \mathrm{~g} / \mathrm{L}$ while the concentration of $\mathrm{HMO}$ in human milk range from 20 to $23 \mathrm{~g} / \mathrm{L}$. More than 60 kinds of BMO have already been identified (Pacheco et al., 2014). The composition of sialylated BMO compounds reaches $70 \%$, whereas it is $20 \%$ for HMOs (Kelly et al., 2013). They are constructed from a lactose heart, but can also be constructed from a lactosamine heart that human milk does not contain. Sialic acids are two in number: Nacetylneuraminic acid (NeuAc) and $\mathrm{N}$-glycolylneuraminic acid (NeuGc). Out of the $70 \%$ of sialylated BMO, $5 \%$ contain NeuGc. Monomers of BMO are: glucose, galactose (Gal), N-acetylglucosamine (GlcNAc), fucose (Fuc), NeuAc and NeuGc. The NeuGc is not found in human milk, HMOs being highly fucosylated $(70 \%)$, while in bovine milk, fucosilation is potentially possible but in very small quantities. The amount of fucose is estimated to be less than $0.01 \%$ (Tao et al., 2008).

BMO could represent an inexpensive and effective source of prebiotic. Indeed, the cheese industry produce whey in large quantities and whey is naturally rich in BMO. The structure of BMO, because of its ramifications, is much closer to HMO than FOS or linear structure. As a result, profits on the host health may be improved (Mehra et al., 2014; Zivkovic et Barile, 2011). In addition they enhance the growth of bifidobacteria, inhibit the attachment of pathogens to the intestinal mucosa and modulate immunity (Urashima et al., 2013).

The polymerization degree of BMOs is lower than that of the HMOs, but the $\beta$-glycosidic bonds of galactose and $\mathrm{N}$ - acetylglucosamine to lactose are similar. Those bonds, like those of HMOs, protect BMO from human digestive enzymes. Indeed, the host has only the ability to digest the glycosidic bond of lactose. The fact that there are more sialylated BMO (N-acetylneuraminic acid and N-lylneuraminic acid) probably affects the bioavailability of BMO (Chichlowski et al., 2011). A hypothesis about the possible mechanisms comparing to HMO is presented in Fig. 1(c).

An interesting study from Meli and coll (2014) assessed the use of a commercial infant formula supplemented with
BMOs instead of lactose. This is the first study reporting the use of BMOs in infant formula. Two formulas were tested: one containing BMOs (Formula 1) and another one containing BMOs and the probiotics B. longum B1999 and Lactobacillus rhamnosus LPR (Formula 2). Both formulas were able to induce changes in infant microbiota by increasing total number of bifidobacteria. No significant difference were observed between Formula 1 or 2 suggesting that with the bifidobacteria concentrations used in this study, the BMOs exerted a more influent effect on total bifidobacteria counts. However, total lactobacilli were not influenced by those formulas.

\section{Conclusion}

Recent progress in molecular biology allowed considerable evolution of the knowledge about bifidobacteria. The study of phylogeny within a specific microbial taxon using only one molecular marker is limited today considering the tremendous progress in molecular biology. Several alternatives approaches were described using $16 \mathrm{~S}$ rRNA gene associated with several housekeeping genes. Those approaches allowed a significant increase of discrimination. Recent studies about bifidobacteria allowed to obtain an Average Nucleotide Identity (ANI) using whole genome sequencing of 48 taxa of bifidobacteria. Using that method, the taxonomy of bifidobacteria could be refined with the recognition of only 34 species of bifidobacteria instead of 39. Indeed, some pairs presented a ANI of $97 \%$ such as B. stercoris, B. coryneforme, B. $\mathrm{ka}$ shiwanohense and $B$. saeculare comparing to respectively B. adolescentis, B. indicum, B. catenulatum and B. gallinarum/B. pullorum). On the other hand, the $B$. pseudolongum subsp. pseudolongum and B. pseudolongum subsp. globosum pair presented an ANI below 94\% and should therefore be reclassified into two different species.

In addition, a better knowledge of the genome of bifidobacteria allows a better understanding of the mechanisms involved in complex carbohydrate metabolism. For example, a co-evolution between HMO present in the microbiota and bifidobacteria able to use them can be identified in the genome. Some bifidobacteria clusters are more particularly adapted for HMO catabolism and that cluster seems to have lost genes encoding glycosyl hydrolases involved in the degradation of plant polysaccharides.

The metabolism of complex sugars such as inuline, fructo-oligosaccharides, galacto-oligosaccharides, human milk oligosaccharides and bovine milk oligosaccharides is well known for several species such as B. longum subsp. 
infantis, B. bifidum and B. breve. The genome of other species of bifidobacteria originated from human and animal demonstrates a remarkable enrichment in genes involved in the metabolism of a wide variety of complex polysaccharides. Those species should be further tested to confirm their potential to metabolize complex oligosaccharides in vitro and in vivo.

\section{References}

1. Adamberg, S., Sumeri, I., Uusna, R., Ambalam, P., Kondepudi, K. K., Adamberg, K., Wadstrom, T., and Ljungh, A. (2014) Survival and synergistic growth of mixed cultures of bifidobacteria and lactobacilli combined with prebiotic oligosaccharides in a gastrointestinal tract simulator. Microb. Ecol. Health Dis. 25, doi: 10.3402.

2. Arrieta, M. C., Stiemsma, L. T., Amenyogbe, N., Brown, E. M., and Finlay, B. (2014) The intestinal microbiome in early life: health and disease. Front. Immunol. 5, 427.

3. Barile, D. and Rastall, R. A. (2013) Human milk and related oligosaccharides as prebiotics. Curr. Opin. Biotechnol. 24, 214-219.

4. Bosscher, D., Van Loo, J., and Franck, A. (2006) Inulin and oligofructose as prebiotics in the prevention of intestinal infections and diseases. Nutr. Res. Rev. 19, 216-226.

5. Bottacini, F., Ventura, M., van Sinderen, D., and O'Connell Motherway, M. (2014) Diversity, ecology and intestinal function of bifidobacteria. Microb. Cell. Fact. 13, S4.

6. Cardelle-Cobas, A., Corzo, N., Olano, A., Pelaez, C., Requena, T., and Avila, M. (2011) Galactooligosaccharides derived from lactose and lactulose: influence of structure on $\mathrm{Lac}$ tobacillus, Streptococcus and Bifidobacterium growth. Int. J. Food Microbiol. 149, 81-87.

7. Chichlowski, M., German, J. B., Lebrilla, C. B., and Mills, D. A. (2011) The influence of milk oligosaccharides on microbiota of infants: opportunities for formulas. Ann. Rev. Food Sci. Technol. 2, 331-351.

8. Delcenserie, V., Gavini, F., China, B., and Daube, G. (2011) Bifidobacterium pseudolongum are efficient indicators of animal fecal contamination in raw milk cheese industry. $B M C$ Microbiol. 11, 178.

9. Delcenserie, V., Taminiau, B., Gavini, F., de Schaetzen, M. A., Cleenwerck, I., Theves, M., Mahieu, M., and Daube, G. (2013) Detection and characterization of Bifidobacterium crudilactis and $B$. mongoliense able to grow during the manufacturing process of French raw milk cheeses. BMC Microbiol. 13, 239.

10. Delétoile, A., Passet, V., Aires, J., Chambaud, I., Butel, M. J., Smokvina, T., and Brisse, S. (2010) Species delineation and clonal diversity in four Bifidobacterium species as revealed by multilocus sequencing. Res. Microbiol. 161, 82-90.

11. De Vuyst, L. and Leroy, F. (2011) Cross-feeding between bifidobacteria and butyrate-producing colon bacteria explains bifidobacterial competitiveness, butyrate production, and gas production. Int. J. Food Microbiol. 149, 73-80.
12. De Vuyst, L., Moens, F., Selak, M., Rivière, A., and Leroy F. (2013) Summer Meeting 2013: growth and physiology of bifidobacteria. J. Appl. Microbiol. 116, 477-491.

13. Di Gioia, D., Aloisio, I., Mazzola, G., and Biavati, B. (2014) Bifidobacteria: their impact on gut microbiota composition and their applications as probiotics in infants. Appl. Microbiol. Biotechnol. 98, 563-577.

14. Garrido, D., Ruiz-Moyano, S., Jimenez-Espinoza, R., Eom, H. J., Block, D. E., and Mills, D. A. (2013) Utilization of galactooligosaccharides by Bifidobacterium longum subsp. infantis isolates. Food Microbiol. 33, 262-270.

15. German, J. B., Freeman, S. L., Lebrilla, C. B., and Mills, D. A. (2008) Human milk oligosaccharides: evolution, structures and bioselectivity as substrates for intestinal bacteria. Nestle Nutr. Workshop Ser. Pediatr. Program. 62, 205-218.

16. Han, K. H., Kobayashi, Y., Nakamura, Y., Shimada, K., Aritsuka, T., Ohba, K., Morita, T., and Fukushima, M. J. (2014) Comparison of the effects of longer chain inulins with different degrees of polymerization on colonic fermentation in a mixed culture of Swine fecal bacteria. Nutr. Sci. Vitaminol. 60, 206-212.

17. Kelly, V., Davis, S., Berry, S., Melis, J., Spelman, R., Snell, R., Lehnert, K., and Palmer, D. (2013) Rapid, quantitative analysis of 3'- and 6'-sialyllactose in milk by flow-injection analysis-mass spectrometry: screening of milks for naturally elevated sialyllactose concentration. J Dairy Sci. 12, 76847691.

18. Konstantinidis, K. T. and Tiedje, J. M. (2005) Genomic insights that advance the species definition for prokaryotes. Proc. Natl. Acad. Sci. U S A. 102, 2567-2572.

19. Lee, J. H. and O'Sullivan, D. J. (2010) Genomic insights into bifidobacteria. Microbiol. Mol. Biol. Rev. 74, 378-416.

20. Lugli, G. A., Milani, C., Turroni, F., Duranti, S., Ferrario, C., Viappiani, A., Mancabelli, L., Mangifesta, M., Taminiau, B., Delcenserie, V., van Sinderen, D., and Ventura M. (2014) Investigation of the evolutionary development of the genus $\mathrm{Bi}$ fidobacterium by comparative genomics. Appl. Environ. Microbiol. 80, 6383-6394.

21. Mehra, R., Barile, D., Marotta, M., Lebrilla, C. B., Chu, C., and German, J. B. (2014) Novel high-molecular weight fucosylated milk oligosaccharides identified in dairy streams. PLoS One. 9, e96040.

22. Meli, F., Puccio, G., Cajozzo, C., Ricottone, G., Pecquet, S., Sprenger, N., and Steenhout, P. (2014) Growth and safety evaluation of infant formulae containing oligosaccharides derived from bovine milk: a randomized, double-blind, noninferiority trial. BMC Pediatr. 14, 306.

23. Milani, C., Lugli, G.A., Duranti, S., Turroni, F., Bottacini, F., Mangifesta, M., Sanchez, B., Viappiani, A., Mancabelli, L., Taminiau, B., Delcenserie, V., Barrangou, R., Margolles, A., van Sinderen, D., and Ventura, M. (2014) Genomic encyclopedia of type strains of the genus Bifidobacterium. Appl. Environ. Microbiol. 80, 6290-6302.

24. Pacheco, A. R., Barile, D., Underwood, M. A., and Mills, D. A. (2014) The impact of the milk glycobiome on the neonate gut microbiota. Annu. Rev. Anim. Biosci. Epub ahead of print 
DOI:10.1146.

25. Rossi, M., Corradini, C., Amaretti, A., Nicolini, M., Pompei, A., Zanoni, S., and Matteuzzi, D. (2005) Fermentation of fructooligosaccharides and inulin by bifidobacteria: a comparative study of pure and fecal cultures. Appl. Environ. Microbiol. 71, 6150-6158.

26. Scholtens, P. A., Goossens, D. A., and Staiano, A. (2014) Stool characteristics of infants receiving short-chain galacto-oligosaccharides and long-chain fructo-oligosaccharides: a review. World J. Gastroenterol. 20, 13446-13452.

27. Sela, D. A., Chapman, J., Adeuya, A., Kim, J. H., Chen, F., Whitehead, T. R., Lapidus, A., Rokhsar, D. S., Lebrilla, C. B., German, J. B., Price, N. P., Richardson, P. M., and Mills, D. A. (2008) The genome sequence of Bifidobacterium longum subsp. infantis reveals adaptations for milk utilization within the infant microbiome. Proc. Natl. Acad. Sci. U S A. 105, 18964-189649.

28. Sela, D. A. and Mills D. A. (2010) Nursing our microbiota: molecular linkages between bifidobacteria and milk oligosaccharides. Trends Microbiol.18, 298-307.

29. Sela, D. A. (2011) Bifidobacterial utilization of human milk oligosaccharides. Int. J. Food Microbiol. 149, 58-64.

30. Smilowitz, J. T., Lebrilla, C. B., Mills, D. A., German, J. B., and Freeman, S. L. (2014) Breast milk oligosaccharides: structure-function relationships in the neonate. Ann. Rev. Nutr. 34, 143-169.

31. Stackebrandt, E., Frederiksen, W., Garrity, G. M., Grimont, P. A., Kämpfer, P., Maiden, M. C., Nesme, X., Rosselló-Mora, R., Swings, J., Trüper, H. G., Vauterin, L., Ward, A. C., and Whitman, W. B. (2002) Report of the ad hoc committee for the re-evaluation of the species definition in bacteriology. Int. J. Syst. Evol. Microbiol. 52, 1043-1047.

32. Stiverson, J., Williams, T., Chen, J., Adams, S., Hustead, D., Price, P., Guerrieri, J., Deacon, J., and Yu Z. (2014) A comparative evaluation of prebiotic oligosaccharides using in vitro cultures of infant fecal microbiome. Appl. Environ. Microbiol. 80, 7388-7397.

33. Tanner, S. A., Chassard, C., Zihler Berner, A., and Lacroix C. (2014) Synergistic effects of Bifidobacterium thermophilum RBL67 and selected prebiotics on inhibition of Salmonella colonization in the swine proximal colon PolyFermS model.
Gut Pathog. 6, 44.

34. Tao, N., DePeters, E. J., Freeman, S., German, J. B., Grimm, R., and Lebrilla, C. B. (2008) Bovine milk glycome. J. Dairy Sci. 91, 3768-3778.

35. Turroni, F., van Sinderen, D., and Ventura, M. (2011) Genomics and ecological overview of the genus Bifidobacterium. Int. J. Food Microbiol. 149, 37-44.

36. Turroni, F., Peano, C., Pass, D. A., Foroni, E., Severgnini, M., Claesson, M. J., Kerr, C., Hourihane, J., Murray, D., Fuligni, F., Gueimonde, M., Margolles, A., De Bellis, G., O'Toole, P. W., van Sinderen, D., Marchesi, J. R., and Ventura, M. (2012) Diversity of bifidobacteria within the infant gut microbiota. PLoS One. 7, e36957.

37. Turroni, F., Duranti, S., Bottacini, F., Guglielmetti, S., Van Sinderen, D., and Ventura, M. (2014) Bifidobacterium bifidum as an example of a specialized human gut commensal. Front. Microbiol. 5, 437.

38. Urashima, T., Taufik, E., Fukuda, K., and Asakuma, S. (2013) Recent advances in studies on milk oligosaccharides of cows and other domestic farm animals. Biosci. Biotechnol. Biochem. 77, 455-466.

39. Van der Meulen, R., Adriany, T., Verbrugghe, K., and De Vuyst L. (2006) Kinetic analysis of bifidobacterial metabolism reveals a minor role for succinic acid in the regeneration of $\mathrm{NAD}+$ through its growth-associated production. Appl. Environ. Microbiol. 72, 5204-5210.

40. Ventura, M., Canchaya, C., Del Casale, A., Dellaglio, F., Neviani, E., Fitzgerald, G. F., and van Sinderen D. (2006) Analysis of bifidobacterial evolution using a multilocus approach. Int. J. Syst. Evol. Microbiol. 56, 2783-2792.

41. Wu, S., Grimm, R., German, J. B., and Lebrilla, C. B. (2011) Annotation and structural analysis of sialylated human milk oligosaccharides. J. Proteome Res. 10, 856-868.

42. Zivkovic, A. M. and Barile, D. (2011) Bovine milk as a source of functional oligosaccharides for improving human health. Adv. Nutr. 2, 284-289.

43. Zoetendal, E. G., Rajilic-Stojanovic, M., and de Vos, W. M. (2008) High-throughput diversity and functionality analysis of the gastrointestinal tract microbiota. Gut 57, 1605-1615.

(Received 2015.2.1/Accepted 2015.2.8) 